\title{
Púrpura de Henoch-Schönlein (Vasculite por IgA) no Adulto
}

\author{
Ana Isabel Gouveia', Leonor Lopes', João Pedro Freitas² \\ 'Interna do Internato Complementar de Dermatologia e Venereologia/Resident, Dermatology and Venereology, Clínica Universitária \\ de Dermatologia, Hospital de Santa Maria (CHLN), Lisboa, Portugal \\ ${ }^{2}$ Assistente Hospitalar Graduado de Dermatologia e Venereologia/Graduated Consultant, Dermatology and Venereology, Clínica \\ Universitária de Dermatologia, Hospital de Santa Maria (CHLN), Lisboa, Portugal
}

RESUMO - A Púrpura de Henoch-Schönlein é uma vasculite de pequenos vasos com deposição de imunocomplexos lgA. Caracteriza-se por púrpura palpável, artralgias/artrite, envolvimento abdominal e renal. A sua etiologia permanece desconhecida, mas vários estímulos têm sido propostos como possíveis fatores desencadeantes, entre eles, agentes infeciosos, fármacos, antígenos tumorais, havendo possível associação a malignidade e doenças autoimunes. Ocorre maioritariamente em crianças, sendo rara nos adultos. Na população pediátrica a doença é, na maioria das vezes, autolimitada, enquanto nos adultos está associada a um pior prognóstico e a um maior número de complicações. Neste artigo abordam-se os principais aspetos da PHS nos adultos, salientando-se as suas particularidades e especificidades.

PALAVRAS-CHAVE - Adulto; Imunoglobulina A; Púrpura Henoch-Schönlein; Vasculite Leucocitoclástica Cutânea.

\section{Henoch-Schönlein Purpura (IgA Vasculitis) in Adults}

ABSTRACT - Henoch-Schönlein purpura is a small vessel vasculitis mediated by the deposition of IgA immune complexes. It is characterized by palpable purpura, arthralgia / arthritis, abdominal and renal involvement. Its etiology remains unknown, but various stimuli have been proposed to trigger this pathology, namely infectious agents, drugs and tumor antigens. There is also a possible association with malignancy and auto-immune diseases. Henoch-Schönlein purpura occurs mostly in children and is rare in adults. In the pediatric population the disease is generally self-limited, whereas in adults it is associated with a worse prognosis and a greater number of complications. This article reviews the main aspects of adult Henoch-Schönlein purpura, highlighting its particularities and specificities.

KEY-WORDS - Adult; Immunoglobulin A; Purpura, Schoenlein-Henoch; Vasculitis, Leukocytoclastic, Cutaneous.

\section{INTRODUÇÃO}

A Púrpura de Henoch-Schönlein (PHS), também designada de Vasculite por IgA (de acordo com a nova classificação do Chapel Hill Consensus Conference de 2012), ' é uma vasculite de pequenos vasos com deposição de imunocomplexos $\lg A .{ }^{2}$ Do ponto de vista histopatológico, pode ser considerada uma forma de vasculite leucocitoclástica que envolve não só a pele, mas também outros orgãos. ${ }^{2}$ Ocorre principalmente em crianças (mais de $90 \%$ dos casos), sendo rara nos adultos. ${ }^{2}$ Caracteriza-se por purpura palpável, artrite/artralgias, queixas gastrointestinais (GI) e envolvimento renal. ${ }^{3}$ Nas crianças a doença é, na maioria das vezes, autolimitada, enquanto nos adultos está associada a um pior prognóstico e a um maior número de complicações, incluindo progressão para insuficiência renal crónica em quase $50 \%$ dos doentes que têm envolvimento renal. ${ }^{2}$ É verdadeiramente uma doença multissistémica e pode resultar em considerável morbidade e mortalidade em particular na forma do adulto que nos propomos rever. ${ }^{2}$

\section{EPIDEMIOLOGIA}

A PHS ocorre frequentemente em crianças. ${ }^{4}$ Tem o pico de incidência na $1^{a}$ e $2^{a}$ década de vida $(90 \%$ com idade inferior a 10 anos). ${ }^{3} \mathrm{~A}$ incidência anual é de 13-20/100 000 em crianças com idade inferior a 17 anos $^{4}$ sendo significativamente menor nos adultos $(1,3-1,4 / 100$ 000) em que a idade
Correspondência: Ana Isabel Gouveia Hospital de Santa Maria, Serviço de Dermatologia Av. Professor Egas Moniz - 1649-035 Lisboa, Portugal E-mail: anaisabelgouveia@hotmail.com
Recebido/Received

23 Maio/23 May 2015

Aceite/Accepted

11 Julho/ 11 July 2016 
média de início é aos 50 anos. $^{3}$ Enquanto nas crianças parece existir uma predominância do sexo masculino de 2:1, nos adultos ambos os sexos são afetados de igual modo. ${ }^{3}$

\section{ETIOLOGIA}

A etiologia da PHS permanece desconhecida. Vários estímulos têm sido propostos como desencadeantes, estimulando a formação de imunocomplexos lgA que são depositados nos pequenos vasos da pele, glomérulos renais e trato $\mathrm{Gl} .{ }^{5}$ Entre eles estão incluídos agentes infeciosos, fármacos e antigénios tumorais. Nos adultos pode haver associação com malignidade e doenças autoimunes. ${ }^{2,5-8}$

A incidência de PHS é maior nos meses de outono e inverno. ${ }^{3}$ A história de infeções (principalmente da orofaringe e respiratórias) recentes ou simultâneas ao aparecimento da vasculite é frequente em crianças mas difícil de comprovar em adultos. Nas crianças os fatores desencadeantes mais comuns são bactérias (Streptococcus spp., Salmonella spp., Shigella spp. e Staphylococcus aureus) e vírus (parvovirus B19, vírus da hepatite $B$, vírus da hepatite $C$ e o vírus da imunodeficiência humana - VIH). Nos adultos os fármacos (antibióticos, inibidores da enzima conversora da angiotensina, antagonistas dos recetores da angiotensina, anti-inflamatórios não esteróides), vacinas, picadas de insetos e determinados alimentos são habitualmente os responsáveis. ${ }^{3,9-11}$ Além destes, têm sido também descritos casos em doentes sob terapêutica com inibidores do fator de necrose tumoral-a (anti-TNF-a), nomeadamente etanercept ${ }^{12}$, adalimumab ${ }^{13}$ e infliximab. ${ }^{14}$

As neoplasias são uma causa conhecida de vasculites. Embora as neoplasias linfo e mieloproliferativas sejam 3 a 5 vezes mais frequentes que os tumores sólidos na globalidade das vasculites, no caso da PHS existe uma maior associação com os tumores sólidos ou suas metástases. ${ }^{5}$ Numa revisão de Zurada et al., ${ }^{5}$ com 31 casos de PHS associada a malignidade, $94 \%$ dos doentes eram do sexo masculino, tinham uma idade média de 64 anos e a maioria era associada a tumores sólidos (61\%), sendo o mais frequente o carcinoma do pulmão de não pequenas células (25\%) seguido do carcinoma da próstata (16\%) e do carcinoma renal (6\%). Dentro das neoplasias hematológicas (39\%), as mais frequentes incluíram o mieloma múltiplo (16\%), o linfoma não-Hodgkin (9\%) e a doença de Hodgkin (6\%). Na maioria dos casos, o diagnóstico de neoplasia é concomitante à PHS, no entanto, pode também preceder ou desenvolver-se após o surgimento da vasculite. Embora a fisiopatologia desta associação permaneça desconhecida, vários mecanismos têm sido propostos e incluem: formação de imunocomplexos circulantes resultantes da expressão de neoantigénios pelas células tumorais; formação de anticorpos com reatividade cruzada com antigénios semelhantes aos das células endoteliais; aumento das citocinas inflamatórias produzidas pelas células neoplásicas; diminuição da clearence de imunocomplexos ou por lesão mecânica direta da parede vascular por trombos ou êmbolos tumorais. ${ }^{5} \mathrm{~A}$ hiperviscosidade sanguínea própria destas patologias pode também causar dano endotelial e aumentar o tempo de contacto facilitador da deposição de imunocomplexos ${ }^{15}$. Além disto, os tratamentos oncológicos, nomeadamente a radioterapia e a quimioterapia, ao destruírem as células malignas, possibilitam a exposição de novos antigénios anteriormente contidos no interior das células podendo desencadear nova resposta imunológica. 5,16 Um outro efeito indireto relaciona-se com a presença de infeções virais ou bacterianas, frequentes em doentes oncológicos particularmente suscetíveis a este tipo de intercorrências. ${ }^{5}$

O aumento da ocorrência entre familiares suporta a possibilidade de predisposição genética. ${ }^{17}$ Vários estudos têm realçado o papel relevante do componente genético na suscetibilidade e gravidade das vasculites, sendo o HLA o principal fator genético implicado. Um estudo realizado por López-Meí́as R. et al. ${ }^{17}$ evidenciou o papel do fenótipo HLA-DRB 1*01 como marcador de suscetibilidade para PHS e adicionalmente o efeito protetor no desenvolvimento desta vasculite em indivíduos portadores do fenótipo HLA-DRB1*03. Num outro estudo realizado pelo mesmo grupo, foi possível demonstrar também uma associação da presença de HLA-B*41:02 com o risco acrescido de PHS na população caucasiana independentemente do seu status HLA-DRB1. ${ }^{18}$

\section{CLÍNICA}

As lesões cutâneas são a manifestação clínica mais comum na PHS, caracterizada por petéquias que evolvem para púrpura palpável (presente em 100\% casos e que pode ser recorrente). ${ }^{2}$ Têm distribuição simétrica e caracteristicamente ocorrem predominantemente nas áreas pendentes (membros inferiores, região glútea e dorso) (Fig. 1A e 1B) podendo por vezes estar associadas a edema dos membros. Bolhas hemorrágicas e necrose cutânea (Fig. 1C) podem ocorrer em $35 \%$ dos doentes. ${ }^{2}$ Artrite/artralgias de caracter transitório, migratório e oligoarticular constitui a segunda manifestação mais comum ( $61 \%$ dos casos). ${ }^{2} \mathrm{O}$ envolvimento $\mathrm{Gl}$ é típico da PHS, ocorre em cerca de $48 \%$ dos doentes e caracteriza-se por vómitos, dor abdominal e hemorragias $\mathrm{Gl}$ secundárias a úlceras (habitualmente na segunda porção do duodeno, íleo e/ou reto). Este envolvimento Gl manifesta-se habitualmente cerca de uma semana após o início das lesões cutâneas, mas em $8 \%$ dos casos pode ser a primeira manifestação clínica. $^{2} \mathrm{O}$ envolvimento renal varia de hematúria microscópica à síndrome nefrótica. ${ }^{19}$ Ocorre em $45-85 \%$ dos doentes, com um risco elevado de progressão para insuficiência renal crónica nos adultos. ${ }^{3}$ Geralmente é detetado nos primeiros dois meses mas, por vezes, pode manifestar-se até seis meses após o início da doença. ${ }^{9}$ Alguns estudos têm sugerido que certos polimorfismos genéticos, como aqueles que envolvem os genes das citocinas (antagonista do recetor da IL-1, IL-1 beta ou (L8), estão associados a um risco aumentado de envolvimento renal. ${ }^{2,20}$

O envolvimento dos genitais externos masculinos ocorre em cerca de 2 a $38 \%$ dos casos de PHS. ${ }^{21,22}$ Os sinais e sintomas incluem edema, dor e equimose, sendo os locais mais frequentemente envolvidos a parede escrotal, o epidídimo, os testículos, o apêndice testicular e o cordão espermático. ${ }^{21}$ Habitualmente as queixas são menos intensas que as 


\section{Caso Clínico}

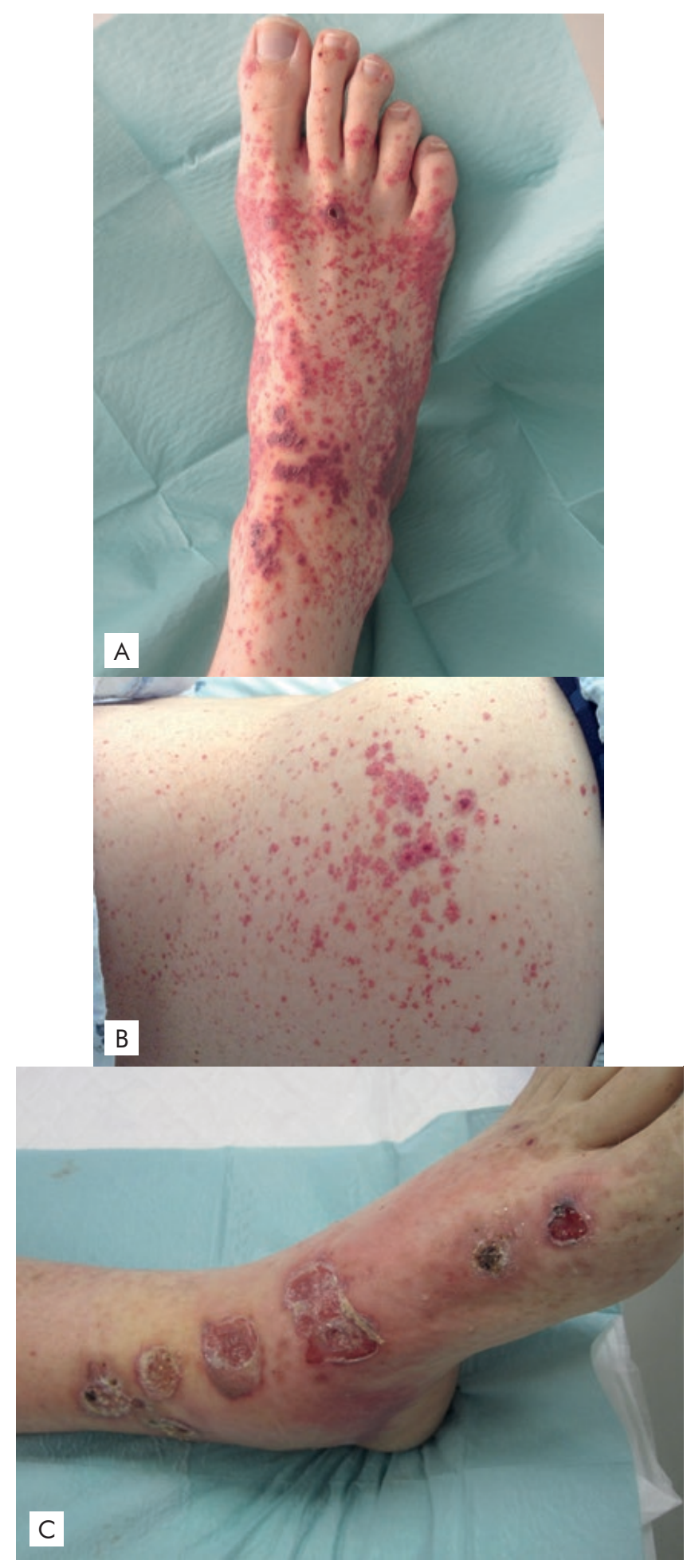

Figura 1 - Manifestações cutâneas de PHS. (A) Purpura palpável no membro inferior. (B) Púrpura palpável na região lombar. (C) Bolhas e áreas de necrose no membro inferior. Imagens referentes a um doente de sexo masculino, de 41 anos de idade, internado no Serviço de Dermatologia do Hospital de Santa Maria com diagnóstico de PHS (de acordo com critérios EULAR/PRINTO/PRES) ${ }^{30}$ e sem fator desencadeante identificado após investigação diagnóstica. observadas na torção testicular, porém, quando as manifestações testiculares ocorrem como manifestação inicial da PHS, o diagnóstico diferencial pode tornar-se mais difícil. ${ }^{21}$

Outras complicações menos frequentes incluem o envolvimento pulmonar, que se manifesta por hemorragia alveolar difusa ou ocasionalmente como pneumonia intersticial ou fibrose intersticial, ${ }^{23}$ o envolvimento do sistema nervoso central e periférico, com possibilidade de ocorrência de convulsões secundárias a vasculite de pequenos vasos cerebrais ${ }^{24} \mathrm{e}$ o envolvimento cardíaco que, apesar de raro, quando ocorre é habitualmente grave e com consequente risco de vida. ${ }^{25}$

\section{DIAGNÓSTICO DIFERENCIAL}

No diagnóstico da PHS é fundamental a exclusão de condições clínicas que a mimetizem e de outras causas de vasculite com manifestações clínicas semelhantes. Devem ser excluídas causas de púrpura trombocitopénica (identificadas pela diminuição na contagem de plaquetas) como a púrpura trombocitopénica imune e a púrpura trombocitopénica trombótica, bem como patologias infeciosas tanto bacterianas como virais, incluindo sépsis. ${ }^{4,11}$ Etiologias secundárias de vasculites como doenças reumatológicas podem também partilhar sintomas com a PHS, destacando-se o lúpus eritematoso sistémico, a artrite reumatóide, a síndrome de Siögren, entre outras. ${ }^{4} \mathrm{~A}$ vasculite por hipersensibilidade induzida por fármacos pode ser particularmente complicada de distinguir da PHS nomeadamente quando esta última está presente na sua forma cutânea isolada. " Outras vasculites de pequenos vasos, como a vasculite urticariforme, a crioglobulinémia mista e as vasculites associados aos ANCA, podem manifestar-se de modo semelhante, podendo ser difícil a sua diferenciação da PHS. ${ }^{4}$ Dado que a dor abdominal é uma manifestação comum na PHS, diferentes causas de abdómen agudo não devem ser esquecidas no diagnóstico diferencial desta vasculite. ${ }^{26,27}$ Uma colheita minuciosa da história clínica e um exame físico atento juntamente com a integração dos achados clínicos, laboratoriais, histológicos e imagiológicos dos exames complementares de diagnóstico, fornecem grande parte da informação necessária para orientar o diagnóstico na maioria dos doentes.

\section{DIAGNOSTICO DEFINITIVO}

Dado não existirem alterações laboratoriais específicas, o diagnóstico da PHS baseia-se na clínica e nos achados histopatológicos. ${ }^{4} \mathrm{~A}$ biópsia cutânea demonstra tipicamente a presença de vasculite leucocitoclástica (Fig. 2A e 2B) e na imunofluorescência direta (IFD) observam-se os depósitos de IgA. Existem evidências que a deposição de IgA não é patognomónica da PHS, podendo ser observada em outras entidades como, por exemplo, nas crioglobulinemias e vasculites por hipersensibilidade secundária a fármacos. ${ }^{28}$ Por outro lado, estão descritos casos de PHS sem deposição de lgA, especulando-se se estes não serão na verdade falsos negativos. Para minimizá-los, as biópsias para IFD devem ser realizadas no bordo de lesões recentes (idealmente com menos de 24 horas) uma vez que as IgAs podem já não ser detetadas 


\section{Caso Clínico}

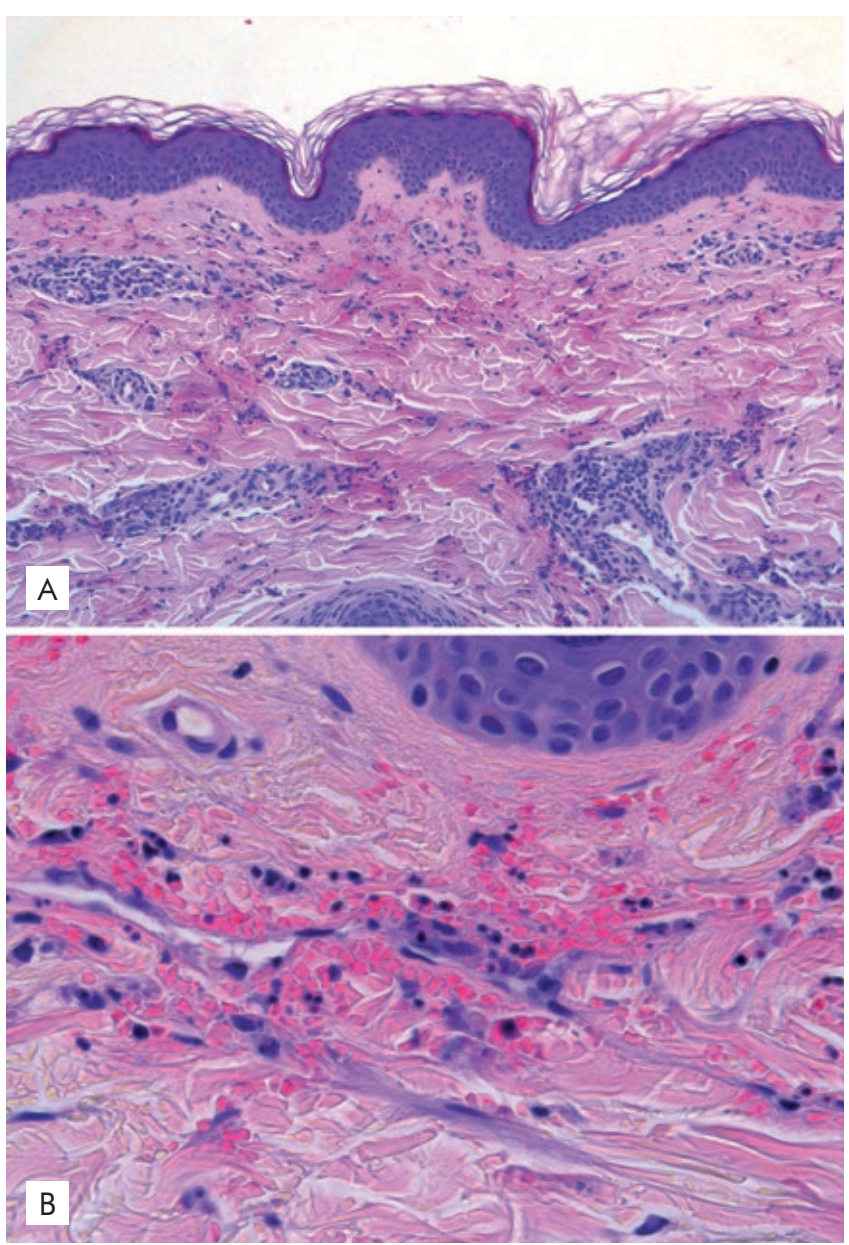

Figura 2 - Exame histopatológico de vasculite leucocitoclástica em doente com PHS. Acentuada extravasão de eritrócitos e leucocitoclasia perivascular e intersticial. (A) HE x100. (B) HE x400. (Exame histopatológico pertencente ao doente ao qual correspondem as imagens da Figura 1)

nas lesões necróticas antigas. ${ }^{28}$ Os resultados de um estudo realizado por Takeuchi et al. ${ }^{29}$ sugerem que a deposição de lgM nas biópsias de lesões purpúricas pode ser um indicador de nefrite em pacientes adultos com PHS, concluindo que estes depósitos de IgM podem estar relacionados com os fatores patogénicos que desencadeiam o envolvimento renal. ${ }^{29}$ A biópsia renal deve ser realizada em caso de diagnóstico incerto ou insuficiência renal grave, tais como a síndrome nefrótica. Outros exames complementares como a endoscopia e/ou colonoscopia desempenham um papel importante no diagnóstico, principalmente em doentes que têm envolvimento $\mathrm{Gl}$ como manifestação inicial. ${ }^{2}$

Em 2010 foram publicados novos critérios da EULAR/ PRINTO/PRES30 (Ankara 2008) para o diagnóstico das vasculites pediátricas, incluindo a PHS (Tabela 1). Os critérios incluem púrpura palpável como critério mandatório, em conjunto com, pelo menos, um dos seguintes achados: dor abdominal difusa, vasculite leucocitoclástica com predominância de depósitos IgA na biópsia cutânea ou biópsia renal, artrite ou artralgia aguda em qualquer articulação e envolvimento renal evidenciado por proteinúria e/ou hematúria. A sensibilidade e a especificidade destes critérios foram, respetivamente, $100 \%$ e $87 \%{ }^{4}$ Apesar de estes critérios ainda não terem sido validados para os adultos, ${ }^{4}$ servem de guia no diagnóstico.

\section{TRATAMENTO}

Na maioria das vezes a PHS é autolimitada e pode exigir pouca ou nenhuma intervenção. ${ }^{19} \mathrm{O}$ repouso é importante para limitar a evolução da púrpura, mas não influencia a evolução do compromisso renal ou Gl. ${ }^{11}$ A analgesia é importante, mas os anti-inflamatórios não esteróides devem ser evitados na presença de envolvimento gástrico e renal. ${ }^{31} \mathrm{No}$ caso de envolvimento renal com proteinúria ligeira a moderada pode ser necessário o uso de inibidores da enzima conversora da angiotensina e, no caso de complicações gastrointestinais, pode ser necessária intervenção cirúrgica. ${ }^{31}$ As terapêuticas específicas mantêm-se controversas e a sua eficácia continua por avaliar, além do que, os estudos que existem, foram realizados maioritariamente na população pediátrica com extrapolação dos resultados para os adultos. ${ }^{31}$

No caso de atingimento cutâneo importante, a colcichina é considerada efetiva em doses de 0,5 a $1 \mathrm{mg} /$ dia. ${ }^{11} \mathrm{~A}$ dapsona é um tratamento conhecido para as dermatoses neutrofílicas especialmente nas caracterizadas por depósitos de IgA. Vários estudos demonstram o seu benefício no atingimento cutâneo e Gl recorrente nos adultos com PHS. 11,32

Foi demonstrado que os leucotrienos estão envolvidos na patogénese da vasculite IgA nas crianças. ${ }^{31}$ Deste modo, o antagonista do recetor dos leucotrienos montelukast foi avaliado como adjuvante no tratamento sintomático com crianças. ${ }^{10} \mathrm{O}$ montelukast foi eficaz na diminuição da púrpura, da dor abdominal, do sangue oculto nas fezes, da artrite, da proteinuria e da hematúria, inibiu as recaídas durante os primeiros três meses após a terapêutica mas não alterou o desfecho de nefrite. ${ }^{31}$ No entanto, não existem estudos controlados nos adultos. ${ }^{31}$

Os corticosteróides são eficazes na diminuição das queixas abdominais e articulares, mas parecem ser ineficazes na resolução das manifestações cutâneas. " Um estudo realizado por Ronkainen et al. ${ }^{33}$ em crianças com PHS, demonstrou que o tratamento precoce com prednisolona foi eficaz na redução dos sintomas abdominais e articulares e na alteração (mas não na prevenção) do envolvimento renal pela doença. O seu efeito na prevenção ou tratamento de nefrite mantém-se controverso. ${ }^{33} \mathrm{Nem}$ todos os doentes com PHS necessitam de corticoterapia precoce, pelo que o tratamento deve visar aqueles com risco elevado de envolvimento renal e sintomas extrarrenais importantes.

Fármacos imunossupressores têm sido usados no tratamento de doentes com PHS grave e refratária. Várias terapêuticas têm sido propostas e incluem, o uso de azatioprina, ${ }^{34}$ ciclosporina $\mathrm{A}^{35}$ e micofenolato de mofetil. ${ }^{36}$ No entanto, a eficácia permanece incerta devido à escassez de estudos controlados." 1 A ciclofosfamida também tem sido 


\section{Caso Clínico}

Tabela 1 - Critérios de Diagnóstico PHS (EULAR/PRINTO/PRES).

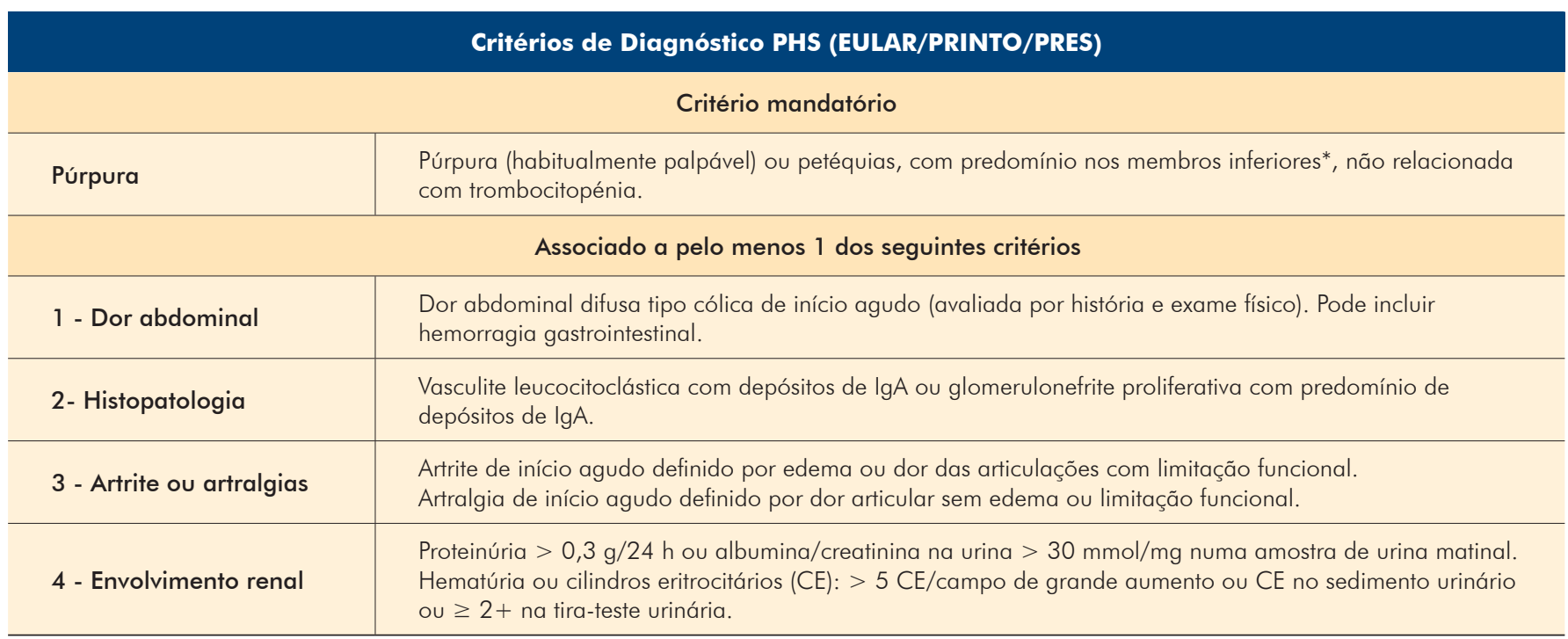

*Se púrpura com distribuição atípica é necessário demonstração histológica de depósitos de lgA.

EULAR, European League Against Rheumatism; PRES, Paediatric Rheumatology European Society, PRINTO, Paediatric Rheumatology International Trials Organisation.

Adaptado de Ozen $\mathrm{S}$ et al. ${ }^{30}$

usada nestes doentes, no entanto, um estudo prospetivo que comparou o uso de prednisona isoladamente e juntamente com ciclofosfamida, revelou que a associação com ciclofosfamida não proporcionou qualquer benefício adicional. ${ }^{37} \mathrm{~A}$ plasmaferese isolada ou em associação com corticosteróides ou imunossupressores tem também sido proposta, bem como o uso de imunoglobulina endovenosa. ${ }^{11,38}$

Perante os resultados pouco expressivos das terapêuticas convencionais, uma nova abordagem com agentes imunomoduladores como o rituximab tem sido proposta. ${ }^{39} \mathrm{O}$ rituximab é um anticorpo monoclonal anti-CD20 usado com sucesso em diferentes formas de vasculite, nomeadamente vasculites associadas aos ANCA e crioglobulinemias porém, neste tipo de vasculite, são ainda necessários mais estudos para avaliar a sua eficácia. ${ }^{31}$ Os candidatos seriam doentes em risco de progressão para doença renal terminal, como o caso daqueles com insuficiência renal na altura do diagnóstico ou doentes com PHS grave com recidiva sob terapêutica com agente poupador de corticosteróides. ${ }^{39}$

\section{PROGNÓSTICO}

A PHS está associada a um bom prognóstico na maioria dos doentes. No entanto, recidivas podem ocorrer até uma década após o início da doença e podem manifestar-se com nefrite de início tardio mesmo no caso de não ter existido doença renal na fase precoce da doença. ${ }^{40}$ Os doentes devem assim manter um seguimento regular a longo prazo.

Os adultos têm com maior frequência envolvimento renal e pior prognóstico. Num estudo realizado por Kang Y, et al. ${ }^{4}$ os autores concluíram que idade avançada, hipertensão, presença de dor abdominal, artralgia, hematúria, proteinúria, síndrome nefrótica, insuficiência renal e anemia na altura do diagnóstico (por análise univariada) são fatores de prognóstico para progressão para insuficiência renal crónica (IRC), identificando como único fator de risco independente (por análise multivariada) a presença de insuficiência renal na altura do diagnóstico.

\section{CONCLUSÃO}

A PHS é uma doença multissistémica e heterogénea. $O$ exame histopatológico da biópsia cutânea confirma a presença de vasculite leucocitoclástica e a IFD permite a observação dos depósitos de $\operatorname{lgA}$ característicos desta patologia. Os adultos têm um pior prognóstico e o desenvolvimento de hematúria pode significar o aparecimento de complicações mais graves como a síndrome nefrítica e nefrótica.

A conduta relativamente ao tratamento é difícil devido à ausência de correlação entre o quadro clínico inicial e a evolução da doença a longo prazo, sendo possível a ocorrência de remissão espontânea nos casos de doença grave, bem como evolução para IRC em doentes com sintomas ligeiros. ${ }^{31}$ Nas vasculites apenas com atingimento cutâneo e articular a terapêutica é essencialmente sintomática. ${ }^{31}$ Nas formas graves e recidivantes o tratamento é controverso, não existindo evidência de que os corticosteróides sistémicos ou os imunossupressores tenham influência na progressão da doença. ${ }^{31}$ A malignidade é uma possibilidade no adulto com PHS e portanto deve ser excluída. Estes doentes devem manter um seguimento regular por um período prolongado, dada a possibilidade de complicações a longo prazo.

\section{Agradecimentos}

Os autores agradecem ao Prof. Doutor Luís Soares de Almeida do Laboratório de Dermatopatologia do Serviço de Dermatologia do Hospital de Santa Maria pelo seu contributo no exame histopatológico. 
Conflitos de interesse: Os autores declaram não possuir conflitos de interesse. Suporte financeiro: $O$ presente trabalho não foi suportado por nenhum subsídio ou bolsa.

Confidencialidade dos dados: Os autores declaram ter seguido os protocolos do seu centro de trabalho acerca da publicação dos dados de doentes.

Conflicts of interest: The authors have no conflicts of interest to declare. Financing Support: This work has not received any contribution, grant or scholarship.

Confidentiality of data: The authors declare that they have followed the protocols of their work center on the publication of data from patients.

\section{REFERÊNCIAS}

1. Jennette JC, Falk RJ, Bacon PA, Basu N, Cid MC, Ferrario, et al. 2012 revised International Chapel Hill Consensus Conference Nomenclature of Vasculitides. Arthritis Rheum. 2013; 65:1-11.

2. Jithpratuck W, Elshenawy Y, Saleh H, Youngberg G, Chi DS, Krishnaswany G. The clinical implications of adult-onset Henoch-Schönelin purpura. Clin Mol Allergy. $2011 ; 9: 9$.

3. Fervenza FC. Henoch-Schönlein purpura nephritis. Int J Dermatol. 2003; 42:170-7.

4. Yang $\mathrm{YH}, \mathrm{Yu} \mathrm{HH}$, Chiang BL. The diagnosis and classification of Henoch-Schönlein purpura: an updated review. Autoimmun Rev. 2014; 13:355-8.

5. Zurada JM, Ward KM, Grossman ME. Henoch-Schönlein purpura associated with malignancy in adults. J Am Acad Dermatol. 2006; 55:S65-70.

6. Mitsui H, Shibagaki N, Kawamura T, Matsue H, Shimada S. A clinical study of Henoch-Schönlein purpura associated with malignancy. J Eur Acad Dermatology Venereol. 2009; 23:394-401.

7. Nishiya K, Oosaki F, Nakamura T, Hashimoto K, Kariya K, Seike M, et al. Rheumatoid arthritis associated with Henoch-Schönlein purpura. Clin Exp Rheumatol. 2000; 18:653-4.

8. Bernardino $V$, Mendes-Bastos P, Rodrigues A, Riso N. IgA vasculitis (formerly Henoch-Schönlein purpura) in an adult with systemic lupus erythematosus. BMJ Case Rep. 2015; 9 .

9. Lu S, Liu D, Xiao J, Yuan W, Zhang X, Zhang J, et al. Comparison between adults and children with Henoch Schönlein purpura nephritis. Pediatr Nephrol. 2015; 30:791-6.

10. Wu SH, Liao PY, Chen XQ, Yin PL, Dong L. Add-on therapy with montelukast in the treatment of Henoch-Schönlein purpura. Pediatr Int. 2014; 56:315-22.

11. Pillebout $E$, Verine J. Purpura rhumatoïde de l'adulte. Rev Med Interne. 2014; 35:372-81.

12. Duffy TN, Genta M, Moll S, Martin PY, Gabay C. Henoch-Schönlein purpura following etanercept treatment of rheumatoid arthritis. Clin Exp Rheumatol. 2006; 24:S106.

13. Rahman FZ, Takhar GK, Roy O, Shepherd A, Bloom SL,
McCartney SA. Henoch-Schönlein purpura complicating adalimumab therapy for Crohn's disease. World J Gastrointest Pharmacol Ther. 2010; 1:119-22.

14. Song Y, Shi YH, He C, Liu CQ, Wang JS, Zhao YJ, et al. Severe Henöch-Schonlein purpura with infliximab for ulcerative colitis. World J Gastroenterol. 2015; $21: 6082-7$.

15. Magro CM, Crowson AN. A clinical and histologic study of 37 cases of immunoglobulin A-associated vasculitis. Am J Dermatopathol. 1999; 21:234-40.

16. Hughes RA, Bottomley DM, Keat AC, Drury A. Henoch-Schönlein purpura occurring in association with carcinoma of the breast. Eur J Med. 1993; 2:310-2.

17. López-Mejías R, Genre F, Pérez BS, Castañeda S, Ortego-Centeno N, Llorca J, et al. HLA-DRB1 association with Henoch-Schönlein purpura. Arthritis Rheumatol. 2014 [in press]

18. López-Mejías R, Genre F, Pérez BS, Castañeda S, Ortego-Centeno N, Llorca J, et al. Association of HLA-B*41:02 with Henoch-Schönlein purpura (IgA Vasculitis) in Spanish individuals irrespective of the HLA-DRB1 status. Arthritis Res Ther. 2015; 17:102.

19. Pillebout E, Thervet E, Hill G, Alberti C, Vanhille P, Nochy D. Henoch-Schönlein purpura in adults: outcome and prognostic factors. J Am Soc Nephrol. 2002; 13:1271-8.

20. Amoli MM, Thomson W, Hajeer AH, Calviño MC, Garcia-Porrua C, Ollier WE, et al. Interleukin 8 gene polymorphism is associated with increased risk of nephritis in cutaneous vasculitis. J Rheumatol. 2002; 29:2367-70.

21. Hara Y, Tajiri T, Matsuura K, Hasegawa A. Acute scrotum caused by Henoch-Schönlein purpura. Int J Urol. 2004; 11:578-80.

22. Modi S, Mohan M, Jennings A. Acute Scrotal Swelling in Henoch-Schönlein purpura: Case Report and Review of the Literature. Urol Case Rep. 2016; 6:9-11.

23. Nadrous HF, Yu AC, Specks U, Ryu JH. Pulmonar involvement in Henoch-Schönlein purpura. Mayo Clin Proc. 2004; 79:1151-7.

24. Fielding RE, Hawkins CP, Hand MF, Heath PD, Davies SJ. Seizures complicating adult Henoch-Schönlein purpura. Nephrol Dial Transplant. 1998; 13:761-2.

25. Lutz HH, Ackermann T, Krombach GA, Gröne HJ, Raven T, Floege J, et al. Henoch-Schönlein purpura complicated by cardiac involvement: case report and review of the literature. Am J Kidney Dis. 2009; 54:e9-15.

26. Choong CK, Beasley SW. Intra-abdominal manifestations of Henoch-Schönlein purpura. J Paediatr Child Health. 1998; 34:405-9.

27. De Castro SM, Joosse P, Unlü C, Steller EP. Henoch-Schönlein disease localized in the appendix. Indian J Pediatr. 2013; 80:1047-9.

28. Linskey KR, Kroshinsky D, Mihm MC Jr, Hoang MP. Immunoglobulin-A-associated small-vessel vasculitis: a 10 year experience at the Massachusetts General Hospital. J Am Acad Dermatol. 2012; 66:813-22.

29. Takeuchi S, Soma Y, Kawakami T. IgM in lesional skin of adults with Henoch-Schönlein purpura is an indication of 


\section{Caso Clínico}

renal involvement. J Am Acad Dermatol. 2010; 63:10269.

30. Ozen S, Pistorio A, lusan SM, Bakkaloglu A, Herlin T, Brik $R$, et al. EULAR/PRINTO/PRES criteria for Henoch-Schönlein purpura, childhood polyarteritis nodosa, childhood Wegener granulomatosis and childhood Takayasu arteritis: Ankara 2008. Part II: Final classification criteria. Ann Rheum Dis. 2010; 69:798-806.

31. Audemard-Verger A, Pillebout E, Guillevin L, Thervet E, Terrier B. IgA vasculitis (Henoch-Shönlein purpura) in adults: Diagnostic and therapeutic aspects. Autoimmun Rev. 2015; 14:579-85.

32. Sarma PS. Dapsone in Henoch-Schönlein purpura. Postgrad Med J. 1994; 70:464-5.

33. Ronkainen J, Koskimies O, Ala-Houhala M, Antikainen $M$, Merenmies J, Rajantie J, et al. Early prednisone therapy in Henoch-Schönlein purpura: a randomized, double-blind, placebo-controlled trial. J Pediatr. 2006; 149:241-7.

34. Foster BJ, Bernard C, Drummond KN, Sharma AK. Effective therapy for severe Henoch-Schönlein purpura nephritis with prednisone and azathioprine: a clinical and histopathologic study. J Pediatr. 2000; 136:370-5.

35. Ohtsuka T. Successful oral cyclosporin therapy for
Henoch-Schönlein purpura nephropathy. J Dermatol. 2009; 36:314-6.

36. Han F, Chen LL, Ren PP, Le JY, Choong PJ, Wang HJ, et al. Mycophenolate mofetil plus prednisone for inducing remission of Henoch-Schönlein purpura nephritis: a retrospective study. J Zhejiang Univ Sci B. 2015; 16:772-9.

37. Pillebout E, Alberti C, Guillevin L, Ouslimani A, Thervet E; CESAR study group. Addition of cyclophosphamide to steroids provides no benefit compared with steroids alone in treating adult patients with severe Henoch Schönlein purpura. Kidney Int. 2010; 78:495-502.

38. Rech J, Fuchs F, Kallert S, Hueber AJ, Requadt C, Manger B, et al. Plasmapheresis therapy in an elderly patient with rapidly progressive Henoch-Schönlein purpura with disseminated organ involvement. Clin Rheumatol. 2007; 26:112-4.

39. Pindi Sala T, Michot J, Snanoudi R, Dollat M, Estève E, Marie B, et al. Successful outcome of a corticodependent Henoch-Schönlein purpura adult with rituximab. Case Rep Med. 2014; 2014:619218.

40. Jauhola $\mathrm{O}$, Ronkainen J, Koskimies O, Ala-Houhala M, Arikoski P, Hölttä T, et al. Outcome of Henoch-Schönlein purpura 8 years after treatment with a placebo or prednisone at disease onset. Pediatr Nephrol. 2012; 27:933-9. 